Spectral and scattering theory, Plenum Publishing Corp., New York, 1998, pp.111-130 (ed.A.G.Ramm)

\title{
RECOVERY OF COMPACTLY SUPPORTED SPHERICALLY SYMMETRIC POTENTIALS FROM THE PHASE SHIFT OF THE S-WAVE
}

\author{
A.G. RAMM \\ Department of Mathematics, Kansas State \\ University, Manhattan, KS 66506-2602, USA
}

\begin{abstract}
It is proved that the phase shift $\delta_{0}(k)$ known for all $k>0$, determines a real-valued locally integrable spherically symmetric potential $q(x),|q(x)| \leq C \exp \left(-c|x|^{\gamma}\right), \gamma>1$, where $\gamma$ is a fixed number, $c$ and $C$ are positive constants. A procedure for finding the unknown potential from the above phase shift is proposed, an iterative process for finding the potential from the above data is described and its convergence is proved.
\end{abstract}

\section{Introduction and the main result.}

The usual statement of the inverse scattering problem on the half-axis consists in finding $q(x) \in$ $L_{1,1}:=\left\{q: q=\bar{q}, \int_{0}^{\infty}(1+x)|q(x)| d x<\infty\right\}$ from the scattering data $\left\{S(k), \lambda_{j}^{2}, s_{j}\right\}$, where $S(k)=\frac{f(-k)}{f(k)}$ is the $S$ - matrix, $f(k)$ is the Jost function, $-\lambda_{j}^{2}$ are the bound states, $\lambda_{j}>0,1 \leq j \leq J, J$ is the number of bound states, and $s_{j}$ are the normalizing constants [Ma], [R1].

The purpose of this paper is to consider the case when it is known a priori that

$$
q(x)=0 \text { for } x \geq a,
$$

or, for some fixed number $\gamma>1$, that

$$
|q(x)| \leq C \exp \left(-c x^{\gamma}\right), \quad \gamma>1, \quad C, \quad c=\text { const }>0,
$$

and to prove that in this case the potential $q(x)$ is uniquely determined by the phase shift known as a function of $k$ on an arbitrary fixed subset of the semiaxis $(0, \infty)$.

This is of interest in physics. Indeed, assuming $q(\mathbf{x}), \mathbf{x} \in \mathbb{R}^{3}$, to be spherically symmetric: $q=q(r)$, $r=|\mathbf{x}|$, one arrives at the one-dimensional inverse scattering problem for the equation

$$
\begin{gathered}
u^{\prime \prime}+k^{2} u-q(x) u=0, \quad x>0, \quad x:=r, \\
u(0, k)=0, \\
u(x, k)=\exp [i \delta(k)] \sin [k x+\delta(k)]+o(1), \quad x \rightarrow \infty,
\end{gathered}
$$

where $\delta(k)$ is the phase shift for the partial wave with angular momentum $\ell=0$. This phase shift is related to $S(k):=\frac{f(-k)}{f(k)}$, the $S$ - matrix, by the formula:

$$
S(k)=\exp [2 i \delta(k)] .
$$

1991 Mathematics Subject Classification. 35R30 PACS 03.65.Nk.

Key words and phrases. inverse scattering, phase shifts, uniqueness theorems, compactly supported potentials.

The author thanks R.Airapetyan for helpful discussions 
The function $f(k)$ is the Jost function, defined below formula $\left(2.7^{\prime}\right)$ and the phase shift $\delta(k)$ has the properties:

$$
\begin{gathered}
\delta(+\infty)=0, \quad \delta(-k)=-\delta(k), \quad k \in \mathbb{R}, \\
f(k)=|f(k)| \exp [-i \delta(k)] .
\end{gathered}
$$

The scattering data in 3D case is the scattering amplitude $A\left(\alpha^{\prime}, \alpha, k\right)$ which for $q=q(r)$ takes the form:

$$
A\left(\alpha^{\prime}, \alpha, k\right)=A\left(\alpha^{\prime} \cdot \alpha, k\right)=\sum_{\ell=0}^{\infty} A_{\ell}(k) Y_{\ell}\left(\alpha^{\prime}\right) \overline{Y_{\ell}(\alpha)}
$$

where $\ell=(\ell, m),-\ell \leq m \leq \ell, A_{\ell}(k)$ do not depend om $m, Y_{\ell}:=Y_{\ell m}$ are the orthonormal in $L^{2}\left(S^{2}\right)$ spherical harmonics, $\alpha^{\prime}$ and $\alpha$ are unit vectors in the direction of scattered and incident waves, $S^{2}$ is the unit sphere in $\mathbb{R}^{3}$,

$$
S_{\ell}(k)=1+\frac{i k A_{\ell}}{2 \pi} \quad \ell=0,1,2, \ldots
$$

and $S(k):=S_{0}(k)$. Therefore the knowledge of $A\left(\alpha^{\prime}, \alpha, k\right)$ for $q=q(r)$ is equivalent to the knowledge of the set $\left\{A_{\ell}(k)\right\}_{\ell=0,1,2, \ldots}$, which is equivalent to the knowledge of the set $\left\{S_{\ell}(k)\right\}_{\ell=0,1,2, \ldots}$. This knowledge implies directly the knowledge of $S(k):=S_{0}(k)$, or, which is equivalent, the knowledge of $\delta(k):=\delta_{0}(k)$, the phase shift. The knowledge of $\delta(k), 0<k<\infty$, does not determine, in general, $\lambda_{j}^{2}$ and $s_{j}, 1 \leq j \leq J$, it determines only $S(k)$ by formula (1.4).

It is therefore of interest to find out for what class of the potentials the phase shift $\delta(k), 0<k<\infty$, determines $q(x)$ (and therefore $\lambda_{j}^{2}$ and $s_{j}$ ) uniquely. This question and related questions were discussed in several earlier papers [Ak], [GW], [GS], [RuSa1-2], where the inverse problem on the whole axis was studied. We note especially paper [GS] which uses the uniqueness of recovery of the potential from the corresponding Weyl's function. In [KS1,2] the phase problem is discussed in connection with inverse scattering. In $[\mathrm{N}]$ the case of inverse scattering with the known bound states and phase shift, but unknown normalizing constants is discussed.

The purpose of this paper is to prove that $\delta(k)$ determines $q(x)$ which satisfies conditions (1.0) or, more generally, $\left(1.0^{\prime}\right)$. Bargmann potentials show that one cannot weaken the rate of decay of $q(x)$ to exponential: uniqueness of the recovery of exponentially decaying $q(x)$ from $\delta(k)$ does not hold. We also emphasize the algorithmic aspect of the inversion procedure, and in this direction the idea used in [RuSa1-2] is useful.

Note that the scattering amplitude known at a fixed $k>0$ for $\alpha^{\prime}$ and $\alpha$ running through some open subsets of $S^{2}$, however small, determines $q(\mathbf{x}), \mathbf{x} \in R^{3}$, uniquely if $q$ is real-valued and compactly supported [R2] (or decays faster than any exponential [RaSt], for example, satisfies $\left(1.0^{\prime}\right)$ ).

Our main results are:

1) uniqueness theorem 1.1,

and

2) reconstruction procedure based on theorems 3.1, 4.1 and 4.2.

In this section we state the uniqueness theorem 1.1, its proof is given in section 2 .

In section 3 an algorithm for recovery of the potential from the phase shift is described.

In section 4 an iterative solution of the inverse problem is justified.

The uniqueness theorem we prove is:

\section{Theorem 1.1.}

If $q(x) \in L_{1,1}$ satisfies condition $\left(1.0^{\prime}\right)$, then $\delta(k)$ determines $q(x)$ uniquely.

\section{Proof of the uniqueness theorem.}

The strategy of the proof is the following.

Step 1. First we construct $f(k)$ uniquely from the given $S(k)=\exp [2 i \delta(k)]$ by solving the Riemann problem:

$$
f(k)=S(-k) f(-k), \quad-\infty<k<\infty,
$$


$f(k)$ is analytic in $\mathbb{C}_{+}$and $f(-k)$ is analytic in $\mathbb{C}_{-}, \mathbb{C}_{+}:=\{k: \operatorname{Im} k>0\}$ is an upper half-plane of the complex $k$-plane, and $\mathbb{C}_{-}$is in the lower one. Index of a function $F(k)$ is denoted ind $F(k)$ and is defined as follows:

$$
\text { ind } F(k):=\frac{1}{2 \pi} \int_{-\infty}^{\infty} d \log F(k)=\frac{1}{2 \pi} \Delta_{-\infty, \infty} \arg F(k),
$$

where $\Delta_{C} \arg F$ is the increment of the argument of $F(k)$ on the contour $C$.

One has: $\operatorname{ind} S(-k)=J \geq 0$ if $f(0) \neq 0$ and $\operatorname{ind} S(-k)=\frac{1}{2}+J$ if $f(0)=0$, so that problem (2.1) is solvable and its general solution bounded at infinity, depends on $J+1$ arbitrary constants [Ga].

However, there is a unique solution to (2.1) with the properties

$$
f(\infty)=1 \text { in } \overline{\mathbb{C}}_{+}, \quad f\left(i \lambda_{j}\right)=0, \quad \dot{f}\left(i \lambda_{j}\right) \neq 0,
$$

where $f(x)$ is analytic in $\overline{\mathbb{C}}_{+}$,

$$
\dot{f}(x, k)=\frac{\partial f}{\partial k}, \quad f^{\prime}(x, k):=\frac{\partial f(x, k)}{\partial x} .
$$

Step 2. If $f(k)$ is found then $i \lambda_{j}, 1 \leq j \leq J$, are uniquely determined as the only zeros of $f(k)$ in the upper half-plane $\mathbb{C}_{+}:=\{k: \operatorname{Im} k>0\}$.

Step 3. From the Marchenko equation

$$
A(x, y)+\int_{x}^{\infty} F(y+t) A(x, t) d t=-F(x+y), \quad y \geq x \geq 0,
$$

where

$$
F(x):=\frac{1}{2 \pi} \int_{-\infty}^{\infty}[1-S(k)] \exp (i k x) d k+\sum_{j=1}^{J} s_{j} \exp \left(-\lambda_{j} x\right),
$$

it follows that the solutions $A_{m}(x, y), m=1,2$, to (2.4), corresponding to the $F_{m}$ which differ only by $s_{j m}, m=1,2$, differ by a term which is of the form $\sum_{j=1}^{J} b_{j}(y) \exp \left(-\lambda_{j} x\right)$.

Therefore the corresponding potentials

$$
q_{m}(x)=-2 \frac{d}{d x} A_{m}(x, x)
$$

differ by a term which decays not faster than an exponential.

Thus, for a unique choice of $s_{j}$ one can get $q(x)$ in (2.6) decaying faster than any exponential function $\exp (-c|x|)$ for any constant $c>0$.

Let us go through these steps in detail:

Step 1. Assume that

$$
h_{+}(k):=h(k)=S(-k) h(-k):=S(-k) h_{-}(k), \quad-\infty<k<\infty,
$$

and

$$
h_{ \pm}(\infty)=1 \text { in } \overline{\mathbb{C}}_{+},
$$

$h(k)$ is an entire analytic function with exactly $J$ simple zeros $i \nu_{j}$ on the imaginary axis in $\mathbb{C}_{+}$, and $\dot{h}\left(i \nu_{j}\right) \neq 0$. Here and below $h_{+}\left(h_{-}\right)$are analytic in $\mathbb{C}_{+}\left(\mathbb{C}_{-}\right)$functions.

Let us prove that $\nu_{j}=\lambda_{j}, 1 \leq j \leq J$, where $i \lambda_{j}$ are the poles of $S(k)$ in $\mathbb{C}_{+}$. Note that if $q(x)$ satisfies (1.0) or $\left(1.0^{\prime}\right)$, then $f(x, k)$, the Jost solution to equation (1.1) (which is the unique solution to (1.1) with asymptotics $f(x, k)=\exp (i k x)+o(1)$ as $x \rightarrow+\infty, k>0)$ and the Jost function $f(k):=f(0, k)$ are entire functions of $k$ and $S(k):=\frac{f(-k)}{f(k)}$ is a meromorphic in $\mathbb{C}$ function of $k$.

Let us first prove the following lemma: 
Lemma 2.1. If $f(i \lambda)=0, \lambda>0$, then $f(-i \lambda) \neq 0$.

Proof. The Wronskian formula [R1, p.279]

$$
f(x, k) f^{\prime}(x,-k)-f^{\prime}(x, k) f(x-k)=-2 i k .
$$

can be continued to $\mathbb{C}$ analytically if $q$ satisfies (1.0) or $\left(1.0^{\prime}\right)$ and at $x=0$ and $k=i \lambda, \lambda>0$, yields, if $f(i \lambda)=0$ :

$$
-f^{\prime}(0, i \lambda) f(0,-i \lambda)=2 \lambda>0 .
$$

Formula (2.9) shows that if $f(i \lambda)=0$, then $f(0,-i \lambda):=f(-i \lambda) \neq 0$, provided that $q(x)$ satisfies (1.0) or $\left(1.0^{\prime}\right)$. that

Since $\delta(k)$ corresponds by the assumption to a $q(x)$ (satisfying $(1.0)$ or $\left(1.0^{\prime}\right)$ ), there exists $f(k)$ such

$$
S(k)=\frac{f(-k)}{f(k)}=\exp [2 i \delta(k)]
$$

and $f(k)$ satisfies $(2.2)$.

We want to prove that any $h(k)$ which satisfies $(2.6),(2.7)$, and which is an entire function of $k$, must be equal to $f(k)$ in $\mathbb{C}_{+}$, and in particular, must have zeros in $\mathbb{C}_{+}$at the points $i \lambda_{j}, 1 \leq j \leq J$, and only at these points.

Let us write (2.6) as

$$
\frac{h_{+}}{f_{+}}:=\frac{h(k)}{f(k)}=\frac{h(-k)}{f(-k)}:=\frac{h_{-}(k)}{f_{-}(k)}, \quad-\infty<k<\infty .
$$

The left-hand side (right-hand side) of $(2.11)$ is analytic in $\mathbb{C}_{+}\left(\mathbb{C}_{-}\right)$except, possibly, at the zeros of $f(k)$ in $\mathbb{C}_{+},\left(\right.$of $f_{-}(k)$ in $\left.\mathbb{C}_{-}\right)$and tends to 1 as $k \rightarrow \infty$ in $\overline{\mathbb{C}}_{+}\left(\overline{\mathbb{C}}_{-}\right)$.

If $f\left(i \lambda_{j}\right)=0, \lambda_{j}>0$, then (2.11) shows that

$$
h\left(i \lambda_{j}\right)=f\left(i \lambda_{j}\right) \frac{h\left(-i \lambda_{j}\right)}{f\left(-i \lambda_{j}\right)}=0,
$$

where we have used Lemma 2.1 , which says that $f\left(-i \lambda_{j}\right) \neq 0$. Since $h(k)$ has exactly $J$ zeros in $\mathbb{C}_{+}$by the assumption, it follows that $\nu_{j}=\lambda_{j}, 1 \leq j \leq J$, and the function $\frac{h_{+}(k)}{f_{+}(k)}:=\frac{h(k)}{f(k)}$ is analytic in $\mathbb{C}_{+}$. Likewise, the function $\frac{h_{-}(k)}{f_{-}(k)}:=\frac{h(k)}{f(k)}$ is analytic in $\mathbb{C}_{-}$. They coincide on the real axis. Therefore each is an analytic continuation of the other. Thus $\frac{h(k)}{f(k)}$ is analytic in $\mathbb{C}$ and equals to 1 at infinity. By Liouville theorem it follows that $\frac{h(k)}{f(k)} \equiv 1$, so $h(k)=f(k), k \in \mathbb{C}$, and Step 1 is completed.

Step 2. In Step 1 we have found the unique entire function $f(k)$ which solves the Riemann problem (2.1), has exactly $J$ purely imaginary simple zeros in $\mathbb{C}$ and tends to 1 at infinity.

Since this solution is analytic in $\mathbb{C}_{+}$, its zeros are uniquely determined. Thus $\delta(k), k \in \mathbb{R}$, determines $\lambda_{j}, 1 \leq j \leq J$, uniquely if $q(x)$ satisfies $(1.0)$ or $\left(1.0^{\prime}\right)$. Step 2 is completed.

Step 3. Let us choose arbitrary numbers $s_{j}>0,1 \leq j \leq J$, and consider the Marchenko equation:

$$
A(x, y)+\int_{x}^{\infty} F(y+t) A(x, t) d t=-F(x+y), \quad y \geq x \geq 0
$$

where $F(x)$ is given by $(2.5)$.

Lemma 2.2. There is at most one choice of $s_{j}$ such that the solution $A(x, y)$ to (2.13) has the property that the potential $q(x)=-2 \frac{d A(x, x)}{d x}$ decays faster than any exponential as $x \rightarrow+\infty$.

Proof. One solution with the above property does exist since we have assumed that $q(x)$ satisfies (1.0) or $\left(1.0^{\prime}\right)$ : for such a potential $A(x, y)$ satisfies (2.13) with the constants $s_{j}, 1 \leq j \leq J$, uniquely determined by $q(x)$, and the corresponding $A(x, y)$ has the property stated in Lemma 2.2 . 
Let us prove that no other choice of $s_{j}$ yields this property.

Consider any other choice $s_{j}^{\prime}>0, s_{j}^{\prime} \neq s_{j}$. Let $B(x, y)$ be the solution to $(2.13)$ with $F(x)$ replaced by $F(x)+M(x)$, where

$$
M(x)=\sum_{j=1}^{J}\left(s_{j}^{\prime}-s_{j}\right) \exp \left(-\lambda_{j} x\right)
$$

Let

$$
a(x, y):=B(x, y)-A(x, y)
$$

Then (2.13) and a similar equation for $B$, namely,

$$
B(x, y)+\int_{x}^{\infty}[M(y+t)+F(y+t)] B(x, t) d t=-F(x+y)-M(x+y),
$$

imply:

$$
a(x, y)+\int_{x}^{\infty} F(y+t) a(x, t) d t=-M(x+y)-\int_{x}^{\infty} M(y+t)[A(x, t)+a(x, t)] d t
$$

Assume that $q$ satisfies (1.0). Then one can prove (see e.g. [R1, p.279]) that

$$
F(y)=0 \text { for } y>2 a, \quad A(x, y)=0 \text { for } y \geq x \geq a
$$

Therefore (2.17) implies:

$$
a(x, x)=-M(2 x)-\int_{x}^{\infty} M(x+t) a(x, t) d t, \quad x>a .
$$

If $s_{j}^{\prime} \neq s_{j}$ for at least one $j, 1 \leq j \leq J$, then $M(2 x)$ decays, as $x \rightarrow+\infty$, not faster than an exponential $\exp \left(-2 \lambda_{j} x\right)$, and the second term in (2.19) equals to

$$
-\sum_{j=1}^{J}\left(s_{j}^{\prime}-s_{j}\right) \exp \left(-\lambda_{j} x\right) \int_{x}^{\infty} \exp \left(-\lambda_{j} t\right) a(x, t) d t=-\sum_{j=1}^{J}\left(s_{j}^{\prime}-s_{j}\right) \exp \left(-\lambda_{j} x\right) b_{j}(x),
$$

where

$$
b_{j}(x):=\int_{x}^{\infty} \exp \left(-\lambda_{j} t\right) a(x, t) d t
$$

For $x>a$, equation $(2.17)$ becomes

$$
a(x, y)=-\int_{x}^{\infty} M(y+t) a(x, t) d t-M(x+y), \quad y \geq x>a,
$$

because of (2.18). Equation (2.22) has a degenerate kernel and can be solved analytically. Let us write $(2.22)$ as

$$
a(x, y)=-\sum_{j=1}^{J} \gamma_{j} \exp \left(-\lambda_{j} y\right) b_{j}(x)-\sum_{j=1}^{J} \gamma_{j} \exp \left(-\lambda_{j} y\right) \exp \left(-\lambda_{j} x\right), \quad y \geq x>a
$$

where

$$
\gamma_{j}:=s_{j}^{\prime}-s_{j}
$$

Then

$$
b_{j}(x)=-\sum_{j=1}^{J} \gamma_{j} a_{i j}(x) b_{j}(x)-\sum_{j=1}^{J} \gamma_{j} a_{i j}(x) \exp \left(-\lambda_{j} x\right) \text {, }
$$


where

$$
a_{i j}(x):=\int_{x}^{\infty} \exp \left[-\left(\lambda_{j}+\lambda_{i}\right) y\right] d y=\frac{\exp \left[-\left(\lambda_{j}+\lambda_{i}\right) x\right]}{\lambda_{j}+\lambda_{i}} .
$$

System (2.25) with matrix (2.26) for large $x$ has diagonally dominant matrix

$$
\delta_{i j}+\gamma_{j} a_{i j}(x)
$$

Therefore system (2.25) is uniquely solvable by iterations and asymptotically one has

$$
b_{i}(x) \sim \sum_{j=1}^{J} \gamma_{j} a_{i j}(x) \exp \left(-\lambda_{j} x\right), \quad x \rightarrow+\infty
$$

From (2.28) and (2.23) it follows that

$$
\begin{aligned}
a(x, x) & \sim-\sum_{j=1}^{J} \gamma_{j} \exp \left(-\lambda_{j} x\right) \sum_{m=1}^{J} \gamma_{m} a_{j m}(x) \exp \left(-\lambda_{m} x\right)-\sum_{j=1}^{J} \lambda_{j} \exp \left(-2 \lambda_{j} x\right) \\
& =-\sum_{j, m=1}^{J} \frac{\gamma_{j} \gamma_{m}}{\lambda_{j}+\lambda_{m}} \exp \left[-2\left(\lambda_{m}+\lambda_{j}\right) x\right]-\sum_{j=1}^{J} \gamma_{j} \exp \left(-2 \lambda_{j} x\right) .
\end{aligned}
$$

Thus, if $q(x)$ satisfies (1.0), that is, $-2 \frac{d A(x, x)}{d x}=0$ for $x>a$, and $0<\lambda_{1}<\lambda_{2}<\ldots \lambda_{n}$, then

$q_{1}(x):=-2 \frac{d B(x, x)}{d x}=-2 \frac{d a(x, x)}{d x}=2 \frac{d}{d x}\left\{\frac{\gamma_{1}^{2}}{2 \lambda_{1}} \exp \left(-4 \lambda_{1} x\right)+\gamma_{1} \exp \left(-2 \lambda_{1} x\right)\right\}[1+o(1)], \quad x \rightarrow+\infty$

It follows from (2.30) that $q_{1}(x)$ decays not faster than $O\left(\exp \left(-2 \lambda_{1} x\right)\right)$ as $x \rightarrow+\infty$ for any choice of $s_{j}^{\prime}$ such that $s_{1}^{\prime}-s_{1} \neq 0$ and if $s_{j}^{\prime}=s_{j}, 1 \leq j \leq i<n$, then $q_{1}(x)$ decays not faster than $O\left(\exp \left(-2 \lambda_{j+1} x\right)\right)$ as $x \rightarrow+\infty$.

Step 3 is completed.

If $q(x)$ satisfies $\left(1.0^{\prime}\right)$ the argument is similar. For such potentials one uses the estimates (see [Ma, p.178, 209]):

$$
\begin{aligned}
&|A(x, y)| \leq c \sigma\left(\frac{x+y}{2}\right), \quad \sigma(x):=\int_{x}^{\infty}|q(s)| d s, \\
&\left|\frac{\partial A\left(x_{1}, x_{2}\right)}{\partial x_{j}}+\frac{1}{4} q\left(\frac{x_{1}+x_{2}}{2}\right)\right|<c \sigma\left(x_{1}\right) \sigma\left(\frac{x_{1}+x_{2}}{2}\right), \quad j=1,2, \\
&|F(2 x)| \leq c \sigma(x) .
\end{aligned}
$$

These estimates show that $F(x), A(x, x)$ and $A(x, y)$, for $y \geq x$, are majorized by $c \sigma(x)$, so that if $q(x)$ satisfies $\left(1.0^{\prime}\right)$ all these functions also satisfy estimates $(2.31)-(2.33)$ with $\sigma(x) \leq c \exp \left(-|x|^{\gamma}\right)$ with $\gamma>1$, where $c=$ const $>0$ denotes different constants.

Integral equation (2.17) yields under the assumption $\left(1.0^{\prime}\right)$ the following equation

$$
a(x, y)+\int_{x}^{\infty} M(y+t) a(x, t) d t=-M(x+y)+O\left(\exp \left(-c|x|^{\gamma}\right)\right)
$$

This equation has a degenerate kernel and can be solved as before. Its solution has the property that $-2 \frac{d a(x, x)}{d x}$ decays as $x \rightarrow+\infty$, not faster than an exponential unless $s_{j}^{\prime}=s_{j}$ for all $j, 1 \leq j \leq J$.

Theorem 1.1 is proved.

Remark 2.1. Theorem 1.1 could be proved differently as follows. First one notices that under the assumptions of Theorem 1.1 the Jost function $f(k)$ admits analytic continuation as an entire function 
on the whole complex plane of $k$. Then one proves that the relation $f\left(i \lambda_{j}\right)=0$ implies $f\left(-i \lambda_{j}\right) \neq 0$. This is done in Lemma 2.1 by using the Wronskian formula which implies

$$
f^{\prime}\left(0, i \lambda_{j}\right) f\left(-i \lambda_{j}\right)=-2 \lambda_{j} \neq 0 .
$$

Next one proves [R1] that the norming constants can be calculated by the formula

$$
s_{j}=i \frac{f\left(-i \lambda_{j}\right)}{\dot{f}\left(i \lambda_{j}\right)}=i \operatorname{Res}_{k=i \lambda_{j}} S(k),
$$

provided that $f(k)$ is an entire function of $k$. Formula (2.36) is derived from the usual formula for $s_{j}$, namely,

$$
s_{j}=\frac{-2 i \lambda_{j}}{f^{\prime}\left(0, i \lambda_{j}\right) \dot{f}\left(i \lambda_{j}\right)}
$$

and from formula (2.35). Therefore the knowledge of the phase shift $\delta(k)$ determines uniquely $f(k)$, and the knowledge of $f(k)$ determines uniquely the numbers $\lambda_{j}$ as the simple zeros of $f(k)$ in the upper half-plane of $k$-plane, and the norming constants by formula (2.36). These data determine $q(x)$ uniquely by the standard theory [Ma].

\section{Algorithm for recovery of the spherically symmetric potentials from the phase-shift of} the $s$-wave.

In this section we assume that $q(x)$ is compactly supported and without loss of generality, that $a=1$. The basic idea is a variant of the one in [R1, p.296] (which is originally proposed in [RuSa2]): one uses the scattering data to get Cauchy data for the transformation kernel $K(x, y)$, and these Cauchy data are used for a derivation of a nonlinear Volterra equation for $q(x)$.

To start with, define $K(x, y)$ as in [Ma] and [R1, p.251]:

$$
\varphi(x, k)=\varphi_{0}+\int_{0}^{x} K(x, y) \varphi_{0} d y, \quad \varphi_{0}:=\varphi_{0}(x, k):=\frac{\sin k x}{k} .
$$

The kernel $K(x, y)$ is the unique solution to the Goursat problem [Ma], [R1, p.251]):

$$
\begin{gathered}
K_{x x}-q(x) K=K_{y y}, \quad-x \leq y \leq x ; \quad K(x, y)=-K(x,-y), \\
K(x, x)=\frac{1}{2} \int_{0}^{x} q(s) d s, \\
K(x, 0)=0,
\end{gathered}
$$

so

$$
q(x)=2 \frac{d K(x, x)}{d x}
$$

If the Cauchy data

$$
\left\{K(1, y), K_{x}(1, y)\right\}_{-1 \leq y \leq 1}
$$

are known, then one gets the following equation for $q(x)$, which is derived in section 4 (see equation (4.5) in section 4):

$$
q=T(q)
$$

where

$$
T(q)=-2 \int_{x}^{1} K(y, 2 x-y) q(y) d y+\xi(x)
$$

and

$$
\xi(x):=2\left[K_{y}(1,2 x-1)+K_{x}(1,2 x-1)\right] .
$$

The kernel $K(x, y)=K(x, y ; q)$ depends on $q(x)$, so (3.7) is a nonlinear Volterra equation. This equation was studied in [R1, pp.299-302]. Let us check that the function $f(x)$ in (3.8), defined by formula (3.9), is uniquely determined by the phase shift $\delta(k)$ if $q(x)$ vanishes for $x>1$. 
Theorem 3.1. The functions $K(1, y)$ and $K_{x}(1, y)$ for $-1 \leq y \leq 1$ are uniquely determined by $\delta(k)$ if $q(x)=0$ for $x>1$.

Proof of Theorem 3.1. In the course of the proof it will be shown how to calculate $K(1, y)$ and $K_{x}(1, y)$ from the data $\delta(k)$. Let us recall that if $q(x)=0$ for $x>1$, then $\delta(k)$ determines uniquely the Jost function $f(k)$ as the unique solution to the Riemann problem (2.1), namely the solution which satisfies the condition $f(\infty)=1$ in $\overline{\mathbb{C}}_{+}$, and has exactly $n$ simple purely imaginary zeros in $\mathbb{C}_{+}$.

Let us assume that Step 1 in section 2 is completed, that is, $f(k)$ is found from $\delta(k)$. Since the function (3.1) has the asymptotics

$$
\varphi=\frac{|f(k)|}{k} \sin [k x+\delta(k)]+o(1) \text { as } x \rightarrow+\infty,
$$

one concludes that for $x>1$, that is, in the region where $q(x)=0,(3.10)$ implies

$$
\varphi(x)=\frac{|f(k)|}{k} \sin [k x+\delta(k)], \quad x>1 .
$$

In particular, (3.1) and (3.11) imply

$$
\int_{0}^{1} K(1, y) \sin k y d y=-\sin k+|f(k)| \sin [k+\delta(k)]
$$

and

$$
\int_{0}^{1} K_{x}(1, y) \sin k y d y=-K(1,1) \sin k-k \cos k+k|f(k)| \cos [k+\delta(k)] .
$$

Since $\delta(k)$ and $|f(k)|$ are known for all $k>0$, one can take $k=n \pi$, and get

$$
\begin{gathered}
\int_{0}^{1} A(y) \sin (n \pi y) d y=A_{n}, \quad A(y):=K(1, y), \\
\int_{0}^{1} B(y) \sin (n \pi y) d y=B_{n}, \quad B(y):=K_{x}(1, y), \\
A_{n}:=|f(n \pi)| \sin [n \pi+\delta(n \pi)]=|f(n \pi)|(-1)^{n} \sin \delta(n \pi), \\
B_{n}:=-n \pi(-1)^{n}+n \pi|f(n \pi)| \cos [n \pi+\delta(n \pi)]=n \pi(-1)^{n}[-1+|f(n \pi)| \cos \delta(n \pi)] .
\end{gathered}
$$

Note that $\left|A_{n}\right|=O\left(\frac{1}{n}\right)$ as $n \rightarrow \infty$ and, if $q$ is piecewise $C^{1}$, then $\left\{B_{n}\right\}=O\left(\frac{1}{n}\right)$ also. If $q \in L^{2}(0,1)$, then the sequence $B_{n}$ belongs to $\ell^{2}$.

Equations (3.14) and (3.15) determine uniquely both functions $A(y)$ and $B(y)$ since the system of functions $\{\sin (n \pi y)\}_{n=1,2, \ldots}$ is a complete orthonormal system in $L^{2}[0,1]$. In fact, $A(y)$ and $B(y)$ can be written as the Fourier series:

$$
A(y)=2 \sum_{n=1}^{\infty} A_{n} \sin (n \pi y), \quad B(y)=2 \sum_{n=1}^{\infty} B_{n} \sin (n \pi y) .
$$

Theorem 3.1 is proved.

Let us now describe an iterative process for finding the potential $q(x)$ from the data $K(1, y)$ and $K_{x}(1, y)$. In section 4 a version of this iterative process is studied in detail. If $A(y)=K(1, y)$ and $B(y)=K_{x}(1, y)$ are found, then $f(x)$ can be calculated by formula (3.9) and one can try to solve equation (3.8) by an iterative process considered in [RuSa1,2]. Namely, take

$$
q_{0}(x)=\xi(x), \quad q_{n+1}(x):=2 \frac{d K_{n}\left(x, x, q_{n}\right)}{d x},
$$


assume that $q_{n}(x)$ is known and find $K_{n}\left(x, y, q_{n}\right)$ as the unique solution to the problem (3.20)-(3.21):

$$
\begin{gathered}
\frac{\partial^{2} K_{n}\left(x, y ; q_{n}\right)}{\partial x^{2}}-q_{n}(x) K_{n}\left(x, y ; q_{n}\right)=\frac{\partial^{2} K_{n}\left(x, y ; q_{n}\right)}{\partial y^{2}}, \quad-1 \leq-x \leq y \leq x \leq 1 \\
K_{n}(1, y)=A(y), \quad \frac{\partial K_{n}(1, y)}{\partial x}=B(y) .
\end{gathered}
$$

Problem (3.20)-(3.21) is a Cauchy problem for the wave equation. Its solution yields $q_{n+1}(x)$ by the second formula (3.19). Then problem (3.20)-(3.21) is solved with $q_{n+1}(x)$ in place of $q_{n}(x)$ and $K_{n+1}\left(x, y ; q_{n+1}\right)$ (in place of $K_{n}\left(x, y ; q_{n}\right)$ ) is found.

In [RuSa2] a proof of convergence of this iterative process is outlined.

Let us discuss a possible method for finding $\lambda_{j}$ from $\delta(k)$.

Note that

$$
\exp [2 i \delta(k)]=\frac{f(-k)}{f(k)}
$$

and

$$
f(-k)=\overline{f(\bar{k})}, \quad k \in \mathbb{C},
$$

where the bar stands for complex conjugate. Formula (3.23) is an immediate consequence of the reality condition

$$
f(-k)=\overline{f(k)}, \quad-\infty<k<\infty
$$

and of the fact that $f(k)$ is an entire function of $k$.

Indeed, if $f(k)=\sum_{j=0}^{\infty} f_{j} k^{j}, f_{j}$ are constants, then (3.24) implies

$$
f_{j}(-1)^{j}=\overline{f_{j}}, \quad \forall j
$$

that is:

$$
f_{2 m}=\overline{f_{2 m}}:=b_{2 m}, \quad f_{2 m+1}=i b_{2 m+1},
$$

where $b_{m}$ are real numbers, so

$$
f(k)=\sum_{m=0}^{\infty} b_{2 m} k^{2 m}+i \sum_{m=0}^{\infty} b_{2 m+1} k^{2 m+1}
$$

Formula (3.23) follows from (3.27).

Consider the function

$$
F_{S}(x)=\frac{1}{2 \pi} \int_{-\infty}^{\infty}\left[1-\frac{f(-k)}{f(k)}\right] \exp (i k x) d k
$$

Since $q(x)=0$ for $x>a$, it follows (see [R1,p.253]) that $A(0, y)=0$ for $y>2 a$.

In what follows we assume without loss of generality that $a=1$ and $q(x)=0$ for $x>1$. Then it follows that

$$
f(k)=1+\int_{0}^{2} A(0, y) \exp (i k y) d y
$$

Therefore,

$$
f(k)-f(-k)=\int_{0}^{2} A(0, y)[\exp (i k y)-\exp (-i k y)] d y .
$$

Let us assume that $x>2$ and $\lambda_{j} \neq 1, \forall j$. Then, using the residue theorem at the last step, one gets:

$$
\begin{aligned}
F_{S}(x) & =\frac{1}{2 \pi} \int_{-\infty}^{\infty} \frac{d k}{f(k)} \int_{0}^{2} A(0, y)(\exp [i k(x+y)]-\exp [i k(x-y)]) d y \\
& =\int_{0}^{2} d y A(0, y)\left(-\frac{d^{2}}{d x^{2}}+1\right) \frac{1}{2 \pi} \int_{-\infty}^{\infty} \frac{d k}{f(k)\left(1+k^{2}\right)}\left[e^{i k(x+y)}-e^{i k(x-y)}\right] \\
& =\left(-\frac{d^{2}}{d x^{2}}+1\right) \int_{0}^{2} d y A(0, y) i\left(\sum_{j=1}^{J} \frac{e^{-\lambda_{j}(x+y)}-e^{-\lambda_{j}(x-y)}}{\dot{f}\left(i \lambda_{j}\right)\left(1-\lambda_{j}^{2}\right)}+\frac{e^{-(x+y)}-e^{-(x-y)}}{f(i) 2 i}\right) \\
& =i\left(-\frac{d^{2}}{d x^{2}}+1\right) \sum_{j=1}^{J} \frac{e^{-\lambda_{j} x}}{\dot{f}\left(i \lambda_{j}\right)\left(1-\lambda_{j}^{2}\right)} \int_{0}^{2} d y A(0, y)\left[e^{-\lambda_{j} y}-e^{\lambda_{j} y}\right], \quad x>2 .
\end{aligned}
$$


Therefore

$$
F_{S}(x)=-i \sum_{j=1}^{J} \frac{e^{-\lambda_{j} x}}{\dot{f}\left(i \lambda_{j}\right)} \int_{0}^{2} A(0, y)\left(e^{\lambda_{j} y}-e^{-\lambda_{j} y}\right) d y, \quad x>2 .
$$

It follows now that $\lambda_{j}$ can be uniquely determined from the asymptotics of $F_{S}(x)$ as $x \rightarrow+\infty$.

Numerically the problem consists in finding exponents $\lambda_{j}>0,0<\lambda_{1}<\lambda_{2}<\ldots \lambda_{J}$, from the knowledge of the function

$$
F_{S}(x)=\sum_{j=1}^{J} \gamma_{j} e^{-\lambda_{j} x}, \quad \gamma_{j}:=\frac{-i \int_{0}^{2} A(0, y)\left[e^{\lambda_{j} y}-e^{-\lambda_{j} y}\right] d y}{\dot{f}\left(i \lambda_{j}\right)}
$$

for all $x>0$. The constants $\gamma_{j}$ and $\lambda_{j}$ are not known a priori, and the number $J$ of bound states is known. Indeed, since $f(k):=|f(k)| e^{-i \delta(k)}$, since $\delta(\infty)=0$ by definition, and $\delta(-k)=-\delta(k)$ for real $k$, one has

$$
\begin{aligned}
J & =\operatorname{ind} f(k):=\frac{1}{2 \pi} \Delta_{(-\infty, \infty)} \arg f(k)=-\frac{1}{2 \pi} \Delta_{(-\infty, \infty)} \delta(k)=-\frac{1}{2 \pi}[\delta(+\infty)-\delta(+0)+\delta(-0)-\delta(-\infty)] \\
& =\frac{1}{\pi} \delta(+0), \text { if } f(0) \neq 0,
\end{aligned}
$$

and

$$
\text { ind } f=J+\frac{1}{2}, \quad \text { if } f(0)=0 \text {, since } \dot{f}(0) \neq 0 .
$$

Note that $\operatorname{ind} S(k)=\operatorname{ind} \frac{f(-k)}{f(k)}=\operatorname{ind} \overline{f(k)}-\operatorname{ind} f(k)=-2 \operatorname{ind} f(k)$. Thus

$$
\operatorname{ind} S(k)= \begin{cases}-2 J & \text { if } f(0) \neq 0 \\ -2 J-1 & \text { if } f(0)=0\end{cases}
$$

A method for finding $\lambda_{j}$ from $F_{S}(x)$ is known: it is Prony's method described, for example, in [R5] and used in [R6]-[R10].

The problem of finding $\lambda_{j}$ from $F_{S}(x)$ is an ill-posed one: since the function $e^{-\lambda_{j} x}$ decay rapidly as $x \rightarrow+\infty$, a small noise in the data $F_{S}(x)$ complicates extraction of $\lambda_{j}$ from $F_{S}(x)$ [R7-9].

If $q(x)=0$ for $x>a$, then $F(x)=0$ for $x>2 a$, where $F(x)$ is defined by (2.5), see (2.18). Therefore, one gets:

$$
F_{S}(x)=-\sum_{j=1}^{J} s_{j} e^{-\lambda_{j} x}, x>2 a,
$$

provided that $q=0$ for $x>a$. From (3.36) and (3.32) one concludes that:

$$
\gamma_{j}=-s_{j}
$$

Therefore formula (2.36) gives another proof, a short one, of Theorem 1.1. Indeed, if $S(k)$ is known, and $q=0$ for $x>a$ then the asymptotics of the right-hand side of (2.36) as $x \rightarrow+\infty$ determines uniquely the numbers $s_{j}, \lambda_{j}$ and $J$.

\section{An iterative solution of the inverse problem.}

In this section we derive equation (3.7) and study its iterative solution.

To derive (3.7), following the Riemann method, let us integrate (3.2) over the triangle $D$ bounded by the straight lines $s=1, t-x=s-x, t-x=x-s$. This yields

$$
\int_{D} q(s) K(s, t) d s d t=\int_{D}\left(K_{s s}-K_{t t}\right) d s d t=\int_{\partial D} K_{s} d t+K_{t} d s:=I,
$$

where Green's formula was used at the last step, $\partial D$ is the boundary of $D$ which is passed counterclockwise. Denote the vertices of the triangle by $A=(1,1), B=(1,2 x-1), C=(x, x)$. The line $C A$ has the 
equation $t=s$, the line $C B$ has the equation $t-x=x-s$, and the line $B A$ has the equation $s=1$. The integral $I$ in (4.1) can be written as

$$
\begin{aligned}
I & =\int_{2 x-1}^{1} K_{s}(1, t) d t-\int_{x}^{1} d_{s} K(s, 2 x-s)-\int_{x}^{1} d_{s} K(s, s) \\
& =\int_{2 x-1}^{1} K_{s}(1, t) d t-K(1,2 x-1)+K(x, x)-K(1,1)+K(x, x) .
\end{aligned}
$$

From (4.1) and (4.2) one gets:

$$
K(x, x)=\frac{1}{2} \int_{x}^{1} d s q(s) \int_{2 x-s}^{s} K(s, t) d t+\frac{K(1,2 x-1)+K(1,1)}{2}-\frac{1}{2} \int_{2 x-1}^{1} K_{s}(1, t) d t .
$$

Similarly, one derives

$$
\begin{aligned}
K(x, y)= & \frac{1}{2} \int_{x}^{1} d s q(s) \int_{y+x-s}^{y-x+s} K(s, t) d t+\frac{K(1, y+x-1)+K(1, y-x+1)}{2} \\
& -\frac{1}{2} \int_{y+x-1}^{y-x+1} K_{s}(1, t) d t, \quad 0 \leq|y| \leq x \leq 1
\end{aligned}
$$

From (4.3) and (3.5) it follows that

$$
q(x)=-2 \int_{x}^{1} q(s) K(s, 2 x-s) d s+2 K_{y}(1,2 x-1)+2 K_{x}(1,2 x-1):=T(q),
$$

which is equation (3.7).

Let us now assume that $\xi(x)$, defined by (3.9), is known, and write equation (4.5) as:

$$
q(x)=A(q)+\xi(x), \quad A(q):=-2 \int_{x}^{1} q(s) K(s, 2 x-s) d s .
$$

Let us consider (4.6) as an equation with the nonlinear operator

$$
T(q):=\xi(x)-2 \int_{x}^{1} q(s) K(s, 2 x-s ; q) d s,
$$

where $K(x, y):=K(x, y ; q)$ is the unique solution to equation (4.4) which we write as

$$
K(x, y)=g(x, y)+\frac{1}{2} \int_{D_{x y}} q(s) K(s, t) d s d t:=g+B(q) K .
$$

Here $D_{x y}$ is the triangle on the $(s, t)$ plane bounded by the straight lines:

$$
s=1, \quad t=y+s-x, \quad t=y-(s-x),
$$

$B(q) K$ is the integral operator in (4.8), and

$$
g:=g(x, y):=\frac{K(1, y+x-1)+K(1, y-x+1)}{2}-\frac{1}{2} \int_{y+x-1}^{y-x+1} K_{s}(1, t) d t, \quad 0 \leq|y| \leq x \leq 1 .
$$

The function $g(x, y)$ is uniquely and numerically efficiently determined by the phase shift $\delta(k)$, as was proved in Theorem 3.1.

Let us assume that

$$
q(x) \in L^{\infty}(0,1) \text { and } \sup _{0 \leq x \leq 1}|q(x)|:=\mathbf{q}
$$


We prove that if $q(x) \in L^{\infty}(0,1)$ is known, then equation (4.8) is uniquely solvable by iterations and its solution $K(x, y)$ is a differentiable function.

Let us define the Banach space $X_{m}$ of $L^{\infty}(0,1)$ functions with the norm

$$
\|q\|_{m}:=\sup _{0 \leq x \leq 1}\left\{e^{-m(1-x)}|q(x)|\right\}
$$

where $m>0$ is a sufficiently large number which we fix later. For any fixed $m>0$ the norm (4.11) is equivalent to $L^{\infty}(0,1)$ norm. The reason for choosing norm (4.11) is simple: for $m>0$ sufficiently large $(m>>1)$ we prove that $T$ considered on a convex closed set

$$
M_{R}:=\left\{q: \sup _{0 \leq x \leq 1}|q(x)| \leq R\right\}
$$

is a contraction map:

$$
\|T(q)-T(p)\|_{m}<\gamma_{m}\|q-p\|_{m}, \quad 0<\gamma_{m}<1, \quad m>>1, \quad p, q \in M_{R} .
$$

Estimate (4.12) alone is not sufficient for the contraction mapping principle to be applicable to equation (4.6) because we did not prove that the map $T$ sends the set $M_{R}$ into itself. However, estimate (4.12) implies that equation (4.6) has a unique solution in $X_{m}$. Indeed, $R>0$ can be chosen arbitrary large, and if equation (4.6) has two solutions, then taking $R$ such that both solutions belong to $M_{R}$, one can use (4.12) to conclude that their difference is zero.

Since the solution of (4.6) is unique, and estimate (4.12) holds, one may try to compute this solution by iterations:

$$
q_{n+1}(x)=T\left(q_{n}\right), \quad q_{0}=0,
$$

where the function $K=K\left(s, t, q_{n}\right)$ in equation (4.6) is the unique solution to (4.8) with $q=q_{n}(s)$, and the functions $\xi(x)$ in (4.6) and $g(x, y)$ in (4.8) do not depend on the index $n$.

In theorem 4.2 an iterative process (4.43) is suggested for a simultaneous computation of $q(x)$ and $K(x, y)$ and convergence of this process is proved. A similar proof can be given for convergence of the process (4.13), but we do not discuss it because one has to calculate the kernel $K(x, y)$ in order to use process (4.13), and process (4.43) calculates both functions, $q(x)$ and $K(x, y)$, simultaneously.

To realize this plan, we first prove the unique solvability of equation (4.8).

Theorem 4.1. Equation (4.8) is uniquely solvable in $L^{\infty}(D)$, where $D:=\{x, y: 0 \leq|y| \leq x \leq 1\}$. The iterative process

$$
K_{n+1}=B(q) K_{n}+g, \quad K_{0}=g,
$$

converges in $X_{m}$ to the unique solution of (4.8).

Theorem 4.1 follows from Lemma 4.2 below.

If $q(x) \in L^{\infty}(0,1)$, then the kernel $K(x, y)=K(x, y ; q)$ of the transformation operator is a differentiable function which is the unique solution to the equation (31) in [R1, p.252]:

$$
K(x, y)=\frac{1}{2} \int_{\frac{x-y}{2}}^{\frac{x+y}{2}} q(s) d s+\int_{\frac{x-y}{2}}^{\frac{x+y}{2}} d s \int_{0}^{\frac{x-y}{2}} q(s+t) K(s+t, s-t) d t .
$$

Equation $\left(4.14^{\prime}\right)$ is uniquely solvable by iterations. This is proved, for example, in [Ma, p.14], where the following estimate is obtained [Ma, p.14]:

$$
|K(x, y)| \leq \frac{1}{2} w\left(\frac{x+y}{2}\right) \exp \left[Q(x)-Q\left(\frac{x+y}{2}\right)-Q\left(\frac{x-y}{2}\right)\right], \quad 0 \leq|y| \leq x
$$

where

$$
w(x):=\max _{0 \leq s \leq x}\left|\int_{0}^{s} q(t) d t\right|, \quad Q(x):=\int_{0}^{x} d s \int_{0}^{s}|q(t)| d t .
$$


Let $0 \leq|y| \leq x \leq 1$, and $\int_{0}^{1}|q(s)| d s \leq \mathbf{q}$, then (4.15) implies

$$
|K(x, y)| \leq c(\mathbf{q})
$$

where $c(\mathbf{q})$ here and below stand for different positive constants.

Estimate (4.17) and equation (4.14') imply :

$$
\int_{-1}^{1}\left|K_{x}(1, t)\right| d t \leq c(\mathbf{q})
$$

Therefore, (4.17) and (4.18), (3.9) and (4.10) yield

$$
|g(x, y)| \leq c(\mathbf{q}) ; \quad|\xi(x)| \leq c(\mathbf{q})
$$

Let us summarize the results:

Lemma 4.1. If $\max _{0 \leq x \leq 1}|q(x)| \leq \mathbf{q}$, then

$$
\max _{0 \leq x \leq 1,0 \leq|y| \leq 1}|g(x, y)| \leq c(\mathbf{q}), \quad \max _{0 \leq x \leq 1}|\xi(x)| \leq c(\mathbf{q}) .
$$

Now let us prove

Lemma 4.2. If $\mathbf{q}<\infty$, then equation (4.8) is uniquely solvable in $L^{\infty}(D)$ by iterations and its solution is

$$
K(x, y)=\sum_{j=0}^{\infty} B^{j}(q) g .
$$

The series (4.21) converges in $C[0,1]$, and

$$
\left|B^{j}(q) g\right| \leq g_{0} \frac{\mathbf{q}^{j}(1-x)^{2 j}}{(2 j) !}, \quad g_{0}:=\max _{0 \leq|y| \leq x \leq 1}|g(x, y)|
$$

Proof of Lemma 4.2. The proof of (4.22) is by induction. Equation (4.21) is an immediate consequence of (4.22). Equations (4.21) and (4.22) imply the unique solvability of (4.8) by iterations. Estimate (4.22) shows that the spectral radius of the linear operator $B(q)$ in $L^{\infty}(D)$ equals zero, so $B(q)$ is a Volterra operator.

So the proof of Lemma 4.2 is complete as soon as (4.22) is proved.

If $j=0$ then (4.22) is obvious. Suppose (4.22) is already proved for $0 \leq i \leq j$, and let us prove it for $i=j+1$. One has, with $B:=B(q)$,

$$
\begin{aligned}
\left|B^{j+1} g\right| & =\left|\frac{1}{2} \int_{D_{x y}} q(s)\left(B^{j} g\right)(s, t) d s d t\right| \leq \frac{1}{2} \int_{x}^{1} d s|q(s)| \int_{x+y-s}^{x-y+s} \frac{g_{0} \mathbf{q}^{j}}{(2 j) !}(1-s)^{2 j} d t \\
& \leq \frac{g_{0} \mathbf{q}^{j+1}}{(2 j) !} \frac{1}{2} \int_{x}^{1} d s(1-s)^{2 j}(2 s-2 x)=\frac{g_{0} \mathbf{q}^{j+1}}{(2 j) !}(1-x)^{2 j+2}\left(\frac{1}{2 j+1}-\frac{1}{2 j+2}\right) \\
& =\frac{g_{0} \mathbf{q}^{j+1}(1-x)^{2 j+2}}{(2 j+2) !} .
\end{aligned}
$$

Thus (4.22) is proved. Lemma 4.2 is proved.

Let us now prove the following lemma: 
Lemma 4.3. If $p, q \in M_{R}$, then

$$
\|T(p)-T(q)\|_{m} \leq \gamma_{m}\|p-q\|_{m}, \quad 0<\gamma_{m}<1
$$

where $\|\cdot\|_{m}$ is defined in (4.11) and $m=m(R)$ is chosen sufficiently large.

Proof of Lemma 4.3. Equations (4.7) and (4.8) form a system of equations for $q(x)$ and $K(x, y)$. Equation (4.8) is a linear with respect to $K(x, y)$ Volterra-type equation, and if $K:=K(x, y ; q)$ is found from (4.8) and is substituted into (4.6), then (4.6) becomes a single nonlinear Volterra-type equation for $q(x)$.

In Lemma 4.2 the solution to (4.8) was investigated.

Let us now prove (4.24). If (4.24) is proved, then it follows that equation (4.6) has at most one solution in $M_{R}$, but since $R>0$ can be taken arbitrary large, this implies that equation (4.6) has at most one solution in $L^{\infty}(0,1)$.

However, since the index $m$ for which $0 \leq \gamma_{m}<1$, depends on $R$, and we do not prove that the map $T$ maps a ball $\left\{q: \sup _{0 \leq x \leq 1}|q(x)| \leq R\right\}$ into itself for a certain $R$, we cannot claim that the contraction mapping principle is applicable and that the iterative process (3.19) - (3.21) converges.

To prove (4.24), consider

$$
T(p)-T(q)=\sum_{j=1}^{\infty} \int_{x}^{1}\left[\left(B^{j}(p) g\right)(s, 2 x-s) p(s)-\left(B^{j}(q) g\right)(s, 2 x-s) q(s)\right] d s .
$$

The idea of the proof is to use the Volterra properties of the operators $B$ and $T$.

One has

$$
p(s)\left(B^{j}(p) g\right)-q(s)\left(B^{j}(q) g\right)(s, 2 x-s)=(p-q)\left(B^{j}(p) g\right)+q(s)\left[B^{j}(p)-B^{j}(q)\right] g .
$$

From (4.22) it follows that

$$
\begin{aligned}
\sup _{0 \leq x \leq 1} & \left\{e^{-m(1-x)} \int_{x}^{1}|p-q|\left|\left(B^{j}(p) g\right)(s, 2 x-s)\right| d s\right\} \\
& \leq \frac{g_{0} \mathbf{p}^{j}}{(2 j) !} \sup _{0 \leq x \leq 1}\left\{e^{-m(1-x)}(1-x)^{2 j} \int_{x}^{1} e^{m(1-s)}|p-q| e^{-m(1-s)} d s\right\} \\
& \leq \frac{g_{0} \mathbf{p}^{j}}{(2 j) !} \sup _{0 \leq x \leq 1}\left\{e^{-m(1-x)}(1-x)^{2 j} \frac{e^{m(1-x)}-1}{m}\right\}\|p-q\|_{m} \\
& \leq \frac{g_{0} \mathbf{p}^{j}}{(2 j) ! m}\|p-q\|_{m}, \quad \mathbf{p}:=\sup _{0 \leq x \leq 1}|p(x)| .
\end{aligned}
$$

Let us use the following representation:

$$
\begin{aligned}
& B^{j}(p)-B^{j}(q)=[B(p)-B(q)] B^{j-1}(p)+B(q)[B(p)-B(q)] B^{j-2}(p) \\
& \quad+B^{2}(q)[B(p)-B(q)] B^{j-3}(p)+\cdots+B^{j-1}(q)[B(p)-B(q)] \\
& \quad=\sum_{i=0}^{j-1} B^{i}(q)[B(p)-B(q)] B^{j-i-1}(p) .
\end{aligned}
$$

Using (4.22) one gets

$$
\left|\left(B^{i}(q)[B(p)-B(q)] B^{j-i-1}(p) g\right)(s, 2 x-s)\right| \leq g_{0} \mathbf{p}^{j-i-1} \mathbf{q}^{i} \int_{s}^{1}|p(\sigma)-q(\sigma)| d \sigma \frac{(1-s)^{2 j-2}}{(2 j-2) !(2 i) !} .
$$


Thus

$$
\begin{array}{rl}
\| \int_{x}^{1} & d s q(s)\left(B^{i}(q)[B(p)-B(q)] B^{j-i-1}(p) g\right)(s, 2 x-s) \|_{m} \\
& \leq \frac{g_{0} \mathbf{p}^{j-i-1} \mathbf{q}^{i}}{(2 j-2 i-2) !(2 i) !} \sup _{0 \leq x \leq 1}\left\{e^{-m(1-x)} \int_{x}^{1} d s \int_{s}^{1}|p(\sigma)-q(\sigma)| d \sigma(1-s)^{2 j-2}\right\} \\
& \leq \frac{g_{0} \mathbf{p}^{j-1} \mathbf{q}^{i}}{(2 j-2 i-2) !(2 i) !} \sup _{0 \leq x \leq 1}\left\{e^{-m(1-x)} \int_{x}^{1} d \sigma|p(\sigma)-q(\sigma)| \int_{x}^{\sigma} d s(1-s)^{2 j-2}\right\} \\
& \leq \frac{g_{0} \mathbf{p}^{j-1} \mathbf{q}^{i}}{(2 j-2 i-2) !(2 i) !} \sup _{0 \leq x \leq 1}\left\{e^{-m(1-x)} \int_{x}^{1} d \sigma|p(\sigma)-q(\sigma)|(1-x)^{2 j-1}\right\} \\
& \leq \frac{g_{0} \mathbf{p}^{j-1} \mathbf{q}^{i}}{m(2 j-2 i-2) !(2 i) !}\|p-q\|_{m} .
\end{array}
$$

From (4.25)-(4.30) one gets

$$
\begin{aligned}
\|T(p)-T(q)\|_{m} & \leq \frac{1}{m} \sum_{j=1}^{\infty}\left(\frac{g_{0} \mathbf{p}^{j}}{(2 j) !}+\sum_{i=0}^{j-1} \frac{g_{0} \mathbf{p}^{j-1} \mathbf{q}^{i}}{(2 j-2 i-2) !(2 i) !}\right)\|p-q\|_{m} \\
& \leq \frac{g_{0}\|p-q\|_{m}}{m}\left(\sum_{j=1}^{\infty} \frac{\mathbf{p}^{j}}{(2 j) !}+\sum_{j=1}^{\infty} \frac{\mathbf{p}^{j-1}}{(2 j-2 i-2) !} \sum_{i=0}^{j-1} \frac{\mathbf{q}^{i}}{(2 i) !}\right) \leq \gamma_{m}\|p-q\|_{m}
\end{aligned}
$$

where

$$
\gamma_{m} \leq \frac{c(\mathbf{p}, \mathbf{q})}{m}
$$

Here

$$
c(\mathbf{p}, \mathbf{q}):=g_{0}\left[e^{\sqrt{\mathbf{p}}}+e^{\sqrt{\mathbf{p}}+\sqrt{\mathbf{q}}}\right], \quad \mathbf{p}:=\sup _{0 \leq x \leq 1}|p(x)|, \quad \mathbf{q}:=\sup _{0 \leq x \leq 1}|q(x)| .
$$

Assume that

$$
\mathbf{p} \leq R, \quad \mathbf{q} \leq R
$$

and choose $m$ such that

$$
\frac{c(\mathbf{p}, \mathbf{q})}{m}<1
$$

Then (4.12) holds for any $p(x)$ and $q(x)$ for which (4.34) holds. Lemma 4.3 is proved.

For exact data $\xi$ and $g$ in (4.7) and (4.8), one knows that the system of equations (4.7), (4.8) has a solution $q(x)$, so there exists a number $R$ such that $\mathbf{q}<R$.

From Lemma 4.3 it follows that this solution is unique in the whole space $L^{\infty}(0,1)$.

Define

$$
u:=\left(\begin{array}{c}
q \\
K
\end{array}\right), h:=\left(\begin{array}{c}
\xi \\
g
\end{array}\right), W(u):=\left(\begin{array}{c}
-2 \int_{x}^{1} q(s) K(s, 2 x-s) d s \\
\frac{1}{2} \int_{D_{x y}} q(s) K(s, t) d s d t
\end{array}\right)
$$

and consider the system of equations (4.6)-(4.8) as an operator equation

$$
u=V(u):=W(u)+h
$$

in the space

$$
L\left(x_{0}\right):=L^{\infty}\left(x_{0}, 1\right) \times L^{\infty}\left(x, y: 0 \leq|y| \leq x \leq 1, x_{0} \leq x \leq 1\right)
$$

of functions with the norm

$$
\|u\|=\sup _{x_{0} \leq x \leq 1}|q(x)|+\sup _{\substack{x_{0} \leq x \leq 1 \\|y| \leq x}}|K(x, y)|
$$


Lemma 4.4. Let $\|h\| \leq R$ and let $R_{1}>R$ be an arbitrary number. Then the map $V$ maps the ball $B\left(R_{1}\right):=\left\{u: u \in L\left(x_{0}\right),\|u\| \leq R_{1}\right\}$ into itself and is a contraction map on $B\left(R_{1}\right)$ provided that

$$
1-x_{0}<\min \left(\frac{\frac{8}{5}\left(R_{1}-R\right)}{R_{1}^{2}}, \frac{2}{5 R_{1}}\right):=\mu
$$

Proof. Note that if $a$ and $b$ are positive numbers and $a+b \leq R_{1}$, then $a b \leq \frac{R_{1}^{2}}{4}$. Therefore, using (4.36) and (4.37), one gets:

$$
\|V(u)\| \leq\|h\|+\left(1-x_{0}\right) \frac{R_{1}^{2}}{2}+\left(1-x_{0}\right)^{2} \frac{R_{1}^{2}}{8}<R+\frac{5}{8} R_{1}^{2}\left(1-x_{0}\right)<R_{1},
$$

so $V$ maps $B\left(R_{1}\right)$ into itself if $1-x_{0}<\frac{\frac{8}{5}\left(R_{1}-R\right)}{R_{1}^{2}}$. Inequality (4.39) holds under weaker that (4.38) assumption. The full strength of (4.38) is used below, in formula (4.40).

Using (4.36), one obtains:

$$
\begin{aligned}
\|V(u)-V(\tilde{u})\|= & \\
& \|W(u)-W(\tilde{u})\| \\
& \leq \sup _{x_{0} \leq x \leq 1}\left\{2 \int_{x}^{1}|q(s)-\tilde{q}(s)||K(s, 2 x-s)| d s+2 \int_{x}^{1}|\tilde{q}(s) \| K-\tilde{K}| d s\right. \\
& \left.+\frac{1}{2} \int_{x}^{1} d s|q-\tilde{q}| \sup _{|t| \leq s}|K(s, t)|(s-x)+\frac{1}{2} \int_{x}^{1} d s|\tilde{q}(s)| \sup _{|t| \leq s}|K(s, t)-\tilde{K}(s, t)|(s-x)\right\} \\
& \leq\|u-\tilde{u}\|\left(1-x_{0}\right)\left[2 R_{1}+\frac{1}{2} R_{1}\right]=\frac{5}{2} R_{1}\left(1-x_{0}\right)\|u-\tilde{u}\|,
\end{aligned}
$$

so $V$ is a contraction on $B\left(R_{1}\right)$ if (4.38) holds. Lemma 4.4 is proved.

From Lemma 4.4 it follows that the system (4.6)-(4.8), or the equivalent system (4.36), is uniquely solvable by iterations for any $h \in B(R)$. The solution to this system belongs to $B\left(R_{1}\right)$ if condition (4.38) holds. This means that the iterative process defines the unique solution $\{\tilde{q}(x), \tilde{K}(x, y)\}$ for $x_{0} \leq x \leq 1$, $1-x_{0}<\mu,|y| \leq x$.

Since the pair $\{q(x), K(x, y)\}$ solves the system (4.6)-(4.8), we conclude that $\tilde{q}=q(x), \tilde{K}=K(x, y)$, where $q(x)$ and $K(x, y)$ is the pair underlying the exact data $\{\xi, g\}$, that is, $q(x)$ is the original potential which generated the data $\delta(k)$ and, therefore, the data $\{\xi, g\}$, and the transformation kernel $K(x, y)=$ $K(x, y ; q)$.

Define now $\left\{\xi_{0}, g_{0}\right\}$, where

$$
\begin{gathered}
\xi_{0}:=2\left[K_{y}\left(x_{0}, 2 x-x_{0}\right)+K_{x}\left(x_{0}, 2 x-x_{0}\right)\right], \\
g_{0}:=\frac{K\left(x_{0}, y+x-x_{0}\right)+K\left(x_{0}, y-x+x_{0}\right)}{2}-\frac{1}{2} \int_{y+x-x_{0}}^{y-x+x_{0}} K_{s}\left(x_{0}, t\right) d t .
\end{gathered}
$$

Data (4.41) and (4.42) are analogous to data (3.9) and (4.10) with $s=1$ being replaced by $s=x_{0}$.

The crucial point now is: since the functions $\tilde{K}(x, y)$ and $\tilde{q}(x)$ which are obtained in the interval $x_{0} \leq x<1$, are the unique functions $q(x)$ and $K(x, y)$ corresponding to the original data $\{\xi, g\}$, one concludes that the data $\left\{\xi_{0}, g_{0}\right\}$ lies in the same ball $B(2 R)$ as the original data $\{\xi, g\}$, because the data $\left\{\xi_{0}, g_{0}\right\}$ depend only on the unique underlying $q(x)$.

Therefore, one can repeat the argument, construct the solution $\{\tilde{q}, \tilde{K}\}, \tilde{q}=q(x), \tilde{K}=K(x, y)$, on the interval $\left(x_{1}, x_{0}\right)$ by iterations, where $x_{0}-x_{1}<\mu$, with the same $\mu$.

Thus, in a finite number of steps one can construct the solution $q(x)$ and the corresponding $K(x, y)$ for any $0 \leq x \leq 1$.

We have proved 
Theorem 4.2. If the original data $\delta(k)$ corresponds to a $q(r) \in L^{\infty}(0,1), q(r)=0$ for $r>1$, then one can uniquely reconstruct $q(r)$ from $\delta(k)$, known for $0<k<\infty$, by the following steps:

1 ) finding the data (3.6) according to Theorem 3.1;

$2)$ solving the system (4.6)-(4.8), or, equivalently, equation (4.37), by iterations:

$$
u_{n+1}=W\left(u_{n}\right)+h, \quad u_{0}=h .
$$

Iterative process (4.43) converges in $L\left(x_{0}\right)$ at least for $x_{0} \leq x \leq 1,1-\mu \leq x_{0} \leq 1$, where $R=\|h\|$. This iterative process yields $q(x)$ and $K(x, y)$ for $x_{0} \leq x \leq 1,|y| \leq x$, and, if one uses the data $\left\{K\left(x_{0}, y\right), K_{x}\left(x_{0}, y\right)\right\}_{0 \leq|y| \leq x_{0}}$, then one can repeat the iterative process on the interval of the same length starting from the point $x_{0}=1-\mu$ with the data $h_{0}:=\left(\begin{array}{l}f_{0} \\ g_{0}\end{array}\right)$, where $\left\{f_{0}, g_{0}\right\}$ are given by (4.41) and (4.42), and obtain $q(x)$ and $K(x, y)$ on the interval $\left(x_{0}-\mu, x_{0}\right)$. In this way one constructs the solution $q(r)$ for all $0<r<1$ in a finite number of steps. 


\section{REFERENCES}

[Ak] Aktosun T., Bound states and inverse scattering for the Schrödinger equation in one dimension, J.Math. Phys 35 (1994), no. 12, 6231-6236.

[Ga] Gakhov, F., Boundary value problems, Pergamon, New York, 1966.

[GS] Gesztesy F. and Simon B.,, Inverse spectral analysis with partial information on the potential I., Helv Phys. Acta 70 (1997), 66-71.

[GW] Grebert, B., Weder, R., Reconstructon of a potential on the line which is a priori known on the half-line, SIAM J. Appl. Math 55 (1995), no. N1, 242-254.

[KS1] Klibanov, M., Sacks, P., Use of partial knowledge of the potential in the phase problem of inverse scattering, J. Comput. Phys 112 (1994), 273-281.

[KS2] _ Phaseless inverse scattering and the phase problem in optics, J. Math. Phys. 33 (1992), 3813-3821.

[Ma] Marchenko, V., Sturm-Liouville operators and applications, Birkhauser, Basel, 1986.

[Mi] Mikhlin, S.,ed., Linear equations of mathematical physics (in Russian), Nauka, Moscow, 1964.

[N] Newton, R., Remarks on scattering theory, Phys.Rev. 101 (1956), 1588-1596.

[R1] Ramm, A.G., Multidimensional Inverse Scattering Problems, Longman, New York, 1992; Expanded Russian edition, MIR, Moscow, 1994.

[R2] - Recovery of the potential from fixed energy scattering data, Inverse Problems 4 (1988), 877-886; 5 (1989), 255.

[R3] - Conditions under which the scattering matrix is analytic, Sov. Physics Doklady 157 (1964), 1073-1075.

[R4] _ Inverse scattering on half-line, J. Math. Anal. App. 133 (1988), no. 2, 543-572.

[R5] _ Scattering by obstacles, D.Reidel, Dordrecht, 1986, pp. 1-442.

[R6] Theoretical and practical aspects of singularity and eigenmode expansion methods, IEEE A-P 28 (1980), no. N6, 897-901.

[R7] Extraction of resonances from transient fields, IEEE A-P Trans. 33 (1985), 223-226.

[R8] Calculation of resonances and their extraction from transient fields, J. Math. Phys. 26 (1985), no. 5, 1012-1020.

[R9]__ On the singularity and eigenmode expansion methods, Electromagnetics 1 (1981), no. N4, 385-394.

[R10] _ Mathematical foundations of the singularity and eigenmode expansion methods, J. Math. Anal. Appl. 86 (1982), 562-591.

[RaSt] Ramm, A.G., Stefanov, P., Fixed-energy inverse scattering for non-compactly supported potentials, Math. Comp. Modelling 18 (1993), no. N1, 57-64.

[RuSa1] Rundell, W., Sacks, P., On the determination of potentials without bound state data, Jour. of Comp. and Appl. Math. 55 (1994), 325-347.

[RuSa2] _ Reconstruction techniques for classical Sturm-Liouville problems, Math. Comp. 58 (1992), 161-183. 